\title{
Solid-state reference electrodes based on carbon nanotubes and polyacrylate membranes
}

\author{
F. Xavier Rius-Ruiz • Anna Kisiel • Agata Michalska • \\ Krzystof Maksymiuk • Jordi Riu • F. Xavier Rius
}

Received: 9 December 2010 / Accepted: 2 January 2011 /Published online: 12 February 2011

(C) The Author(s) 2011. This article is published with open access at Springerlink.com

\begin{abstract}
A novel potentiometric solid-state reference electrode containing single-walled carbon nanotubes as the transducer layer between a polyacrylate membrane and the conductor is reported here. Single-walled carbon nanotubes act as an efficient transducer of the constant potentiometric signal originating from the reference membrane containing the $\mathrm{Ag} / \mathrm{AgCl} / \mathrm{Cl}^{-}$ions system, and they are needed to obtain a stable reference potentiometric signal. Furthermore, we have taken advantage of the light insensitivity of single-walled carbon nanotubes to improve the analytical performance characteristics of previously reported solid-state reference electrodes. Four different polyacrylate polymers have been selected in order to identify the most efficient reservoir for the $\mathrm{Ag} / \mathrm{AgCl}$ system. Finally, two different arrangements have been assessed: (1) a solid-state reference electrode using photopolymerised $n$-butyl acrylate polymer and (2) a thermopolymerised methyl methacrylate: $n$-butyl acrylate (1:10) polymer. The sensitivity to various salts, $\mathrm{pH}$ and light, as well as time of response and stability, has been tested: the best results were obtained using single-walled carbon nanotubes and photo-polymerised $n$-butyl acrylate polymer. Water transport plays an important role in the potentiometric performance of acrylate membranes, so a new screening test method has been developed to qualitatively assess the
\end{abstract}

\footnotetext{
F. X. Rius-Ruiz $\cdot$ J. Riu $(\bowtie) \cdot$ F. X. Rius

Department of Analytical and Organic Chemistry,

Universitat Rovira i Virgili,

Campus Sescelades, C/Marcel·lí Domingo s/n,

43007 Tarragona, Spain

e-mail: jordi.riu@urv.cat
}

A. Kisiel $\cdot$ A. Michalska $\cdot$ K. Maksymiuk

Department of Chemistry, Warsaw University,

Pasteura 1,

02-093 Warsaw, Poland difference in water percolation between the polyacrylic membranes studied. The results presented here open the way for the true miniaturisation of potentiometric systems using the excellent properties of single-walled carbon nanotubes.

Keywords Solid-state reference electrodes · Carbon nanotubes $\cdot$ Polyacrylate membrane $\cdot$ Water transport

\section{Introduction}

Recent advances in potentiometry have led to extremely low detection limits and high selectivity together with important operational features such as portability and simplicity, thus placing this technique at the forefront of the electrochemical methodologies that are able to perform in situ analysis [1]. However, further steps towards the true miniaturisation of potentiometric sensors are necessary so that they can be put to general use. One of these steps is the development of reliable solid-state reference electrodes (SSREs) that allow the construction of a fully miniaturised potentiometric cell including miniaturised indicator and reference electrodes.

The first polymer-based SSRE appeared due to the necessity of finding alternatives to solid-state $\mathrm{Ag} / \mathrm{AgCl}$ pseudo-reference electrodes, which only work at constant sample chloride activity. First liquid junction-free SSRE prototypes only functioned under ideal or extremely welldefined conditions, which reduced the number of applications they could be used for [2, 3]. Later, Lee et al. reported the insensitivity of some polyurethane polymers as membranes for coated-wire type of SSRE [4], but medium- and long-term stability tests were not detailed. These early ideas were subsequently developed by Mamińska et al. [5] and 
Mattinnen et al. [6]. Both groups reported on PVC-based electroneutral membranes capable of being miniaturised and on the use of a solid redox-type transducers $(\mathrm{Ag} / \mathrm{AgCl}$ or conducting polymers) so a better signal stability could be attained in comparison with coated-wire-type SSREs. However, these SSREs rely on the formation of a constant potential at an electroneutral membrane layer, and unfortunately, sample ions and lipophilic species entering the membrane may be the origin of interferences.

An alternative approach is to use conducting polymers (CPs) with suppressed or compensated anion and cation exchange in direct contact with the sample solution [7-9]. However, SSREs based on CPs are directly affected by redox species in solution [10] and show some of the drawbacks inherent in the use of CPs such as the necessity of tuning the doping conditions for different CPs or the marked light sensitivity of some CPs [11], which makes indispensable the use of protective systems that isolate them from changes in ambient light intensity.

The use of a polymeric protective layer containing the $\mathrm{Ag} / \mathrm{AgCl} / \mathrm{Cl}^{-}$ions system or some components of it has been repetitively proposed in order to obtain constant potentials. The approach is not trivial due to the difficulties in preserving constant salts concentration in a solid state, while maintaining the conductive properties of the polymeric layer [12]. Tymecki et al. proposed the miniaturisation of SSREs by encapsulating chloride salts in a screenprinted insulator paste in contact with $\mathrm{Ag} / \mathrm{AgCl}$ ink [13]. The reported stability of this planar SSRE was excellent, but response to $\mathrm{Ag}^{+}, \mathrm{Pb}^{2+}$ and $\mathrm{Fe}^{3+}$ was observed due to the formation of insoluble species at the solid electrolyte/ solution interface. Again, nonresponsive polymer materials showed promising insensitivity when encapsulating the $\mathrm{Ag} /$ $\mathrm{AgCl} / \mathrm{Cl}^{-}$ions system [14], although it has been argued that similar results could be difficult to reproduce in miniaturised configurations [12]. Other lipophilic materials, such as ionic liquids (ILs), have also been proposed for the entrapment of $\mathrm{Ag} / \mathrm{AgCl}$ salts. ILs-SSREs showed promising results, and only the acute tendency of hydrophobic species (e.g. non-ionic species and surfactants) to enter the bulk membrane and the finite solubility of toxic IL in the sample solution hinder their on-field applications [15]. Recently, Kisiel et al. attained a high degree of solidification of the reference system by dispersing the $\mathrm{Ag} / \mathrm{AgCl} / \mathrm{Cl}^{-}$ ions system into strong hardening polymers such as polyvinyl chloride (PVC) or poly ( $n$-butyl acrylate) [10, 16-18]. Alternatively, the solidification of the reference electrolyte on its own has been proposed as a valid alternative for applications in the food industry, where high temperature and pressures are attained during sterilisation [19]. Excessive electrolyte leakage into the sample solution was only ameliorated by optimised ceramic materials acting as liquid junction.
To date, the redox materials $\mathrm{Ag} / \mathrm{AgCl}$ and $\mathrm{CPs}$ have been the only transducers used in SSREs. Conducting polymers perform as excellent redox transducers, but some of them display practical limitations, such as light sensitivity, the formation of a water layer at the interface between the transducer and the polymeric membrane and the formation of unwanted redox reactions [20]. Therefore, new solid-state transducing materials can be of great interest in order to develop novel SSREs with enhanced analytical and operational characteristics. Single-walled carbon nanotubes (SWCNTs) have been shown to be excellent ion-to-electron transducers for allsolid-state ISEs and thus represent an excellent alternative to CPs [21]. Our group recently demonstrated that the transduction performance of SWCNTs is mediated by their low resistance and their large bulk double-layer capacitance [22]. As a result, SWCNTs provide stable and rapid potentiometric responses, are chemically stable, are not affected by light and prevent the formation of undesirable inner water layers at the membrane-transducer interface [23].

The choice of the protective layer which shields the transducer from interferences and creates a constant potential simultaneously is generalised nowadays in SSREs. Moreover, it has been sought as an advantage that the same materials that will constitute the potentiometric cell could be used in both the solid-sate ISE and RE $[5,17$, 24] or that the production method for both electrodes is compatible [13]. Polymeric membranes are largely used today in solid-state ISEs: polyacrylic polymers which have been reported to have better analytical performance characteristics in comparison with plasticised membranes based on its low ion and water diffusion coefficients [25]. Therefore, the use of novel polyacrylic polymers is attractive because it represents new alternatives in the fabrication of SSREs and solid-state potentiometric systems, which could be ultimately miniaturised.

The aim of the present article is to develop a new SSRE containing SWCNTs as ion-to-electron transducer and polyacrylic membranes for encapsulating the $\mathrm{Ag} / \mathrm{AgCl} / \mathrm{Cl}^{-}$ ions system. SWCNTs have been used here for the first time as transducer layer in SSREs in order to improve the analytical performance characteristics of the proposed SSREs with respect to previously reported CPsbased SSREs. Given the excellent properties of polyacrylic polymers, the best reservoir layers have been selected among four different polyacrylic polymers in order to obtain SSREs with excellent analytical performance characteristics. Water percolation through the membrane has been assessed with a novel screening method that allows us a rapid and qualitative water percolation comparison between four different polyacrylic membranes. 


\section{Experimental section}

Reagents

Tetradodecylammonium tetrakis(4-chlorophenyl)borate (ETH500), tetrahydrofuran (THF), 2,2'-azobis(2-methylpropionitrile) (AIBN), methyl methacrylate (MMA), n-butyl acrylate (nBA) monomers, $\mathrm{KCl}, \mathrm{NaCl}$ and $\mathrm{NaNO}_{3}$ were purchased in Selectophore grade from Fluka. Sodium dodecyl sulphate (SDS), cross-linker 1,6-hexanediol diacrylate (HDDA), photoinitiator 2,2-dimethoxy-2-phenylacetophenone (DMPP) and regioregular poly(octylthiophene) (POT) were purchased from Aldrich. $\mathrm{AgCl}$ was precipitated by mixing $3 \mathrm{M} \mathrm{AgNO}_{3}$ and $3 \mathrm{M} \mathrm{KCl}$ solutions. The sediment formed was filtered on a soft filter, washed and dried in oven at $80{ }^{\circ} \mathrm{C}$, for at least $1 \mathrm{~h} . \mathrm{AgCl}$ was stored in a dark place until use. Single-walled carbon nanotubes with $>90 \%$ weight purity were purchased from $\mathrm{HeJi}$, Inc. and subsequently purified and carboxilated using protocols available in the literature [26]. Milli-Q PLUS (Millipore Corporation) water was used to prepare all the aqueous solutions. Sigladur glassy carbon (GC) and $\mathrm{Cu}$ rods with $3 \mathrm{~mm}$ diameter were obtained from HTW (Germany) and RS Components (Spain), respectively.

\section{Electrode fabrication}

The reference electrode was prepared by pre-processing the surface of the distal end of a glassy carbon rod onto which the SWCNTs and the reference membrane were then deposited. The GC distal end was polished using a sheet of abrasive paper (Buehler Carbimet 600/P1200) and subsequently treated using alumina of different grain sizes (30, 5 and $1 \mu \mathrm{m}$ Buehler Micropolish II). SWCNT dispersion was homogenised by tip-sonication for $60 \mathrm{~min}$ (amplitude 60\%, cycle 0.5, Ultraschallprozessor UP200S, Dr. Hielscher); then, carboxylated SWCNTs were deposited onto the distal end of the GC rod by spraying $10 \mathrm{~mL}$ of an aqueous dispersion containing $25 \mathrm{mg}$ of carboxylated SWCNT and $100 \mathrm{mg}$ of SDS. Finally, SDS is removed by heating the electrodes at $300{ }^{\circ} \mathrm{C}$ for $1 \mathrm{~h}$, yielding a $\sim 30 \mu \mathrm{m}$ layer of SWCNTs. Figure 1 displays a schematic representation of the SWCNT-based SSRE. Then, the SWCNTs layer was covered with the reference membranes as described below.

\section{Preparation of polymeric membranes}

Four different polymeric membranes were prepared: thermo-polymerised nBA, thermo-polymerised MMA: $\mathrm{nBA}$, photo-polymerised $\mathrm{nBA}$ and photo-polymerised MMA:nBA. Thermo-polymerised nBA was synthesised as described elsewhere [27]. Thermo-polymerised MMA:nBA

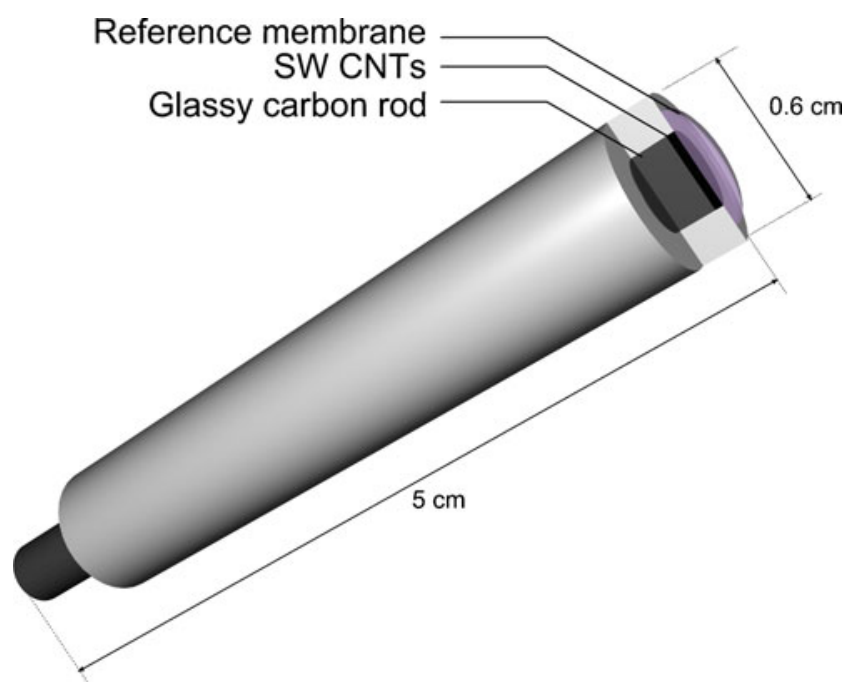

Fig. 1 Schematic representation of the SWCNT-based SSREs

(thermo-MMA:nBA) was synthesised by mixing $500 \mu \mathrm{L}$ of MMA monomer, $4.500 \mu \mathrm{L}$ of nBA monomer and $22 \mathrm{mg}$ of AIBN initiator. The mixture was thoroughly degassed with $\mathrm{N}_{2}$ and heated at $100{ }^{\circ} \mathrm{C}$ for $14 \mathrm{~h}$. Finally, the reaction mixture was heated for $1 \mathrm{~h}\left(100{ }^{\circ} \mathrm{C}\right)$ under low pressure (water pump) to eliminate non-reacted monomers. To prepare the reference membrane cocktail, $200 \mathrm{mg}$ of the chosen polymer, $34 \mathrm{mg}$ of $\mathrm{KCl}, 13 \mathrm{mg}$ of $\mathrm{AgCl}$ (with traces of $\mathrm{Ag}$ ) and $5 \mathrm{mg}$ of ETH500 were dispersed in $1 \mathrm{~mL}$ of THF. One hundred microlitres of the cocktail membrane were drop-cast onto the electrodes and left to dry overnight.

A cross-linking mixture was used to prepare photopolymerised poly( $n$-butyl acrylate) (photo-nBA) membranes which contained $42.7 \mathrm{mg}$ HDDA cross-linker, $457.2 \mathrm{mg}$ DMPP photo-initiator and $3.3 \mathrm{~mL}$ of $n$-butyl acrylate. Photocured reference membrane cocktails were prepared with $180 \mu \mathrm{L}$ of nBA monomer for photo-nBA, or $160 \mu \mathrm{L}$ of nBA monomer and $20 \mu \mathrm{L}$ of MMA monomer for photo-MMA:nBA, plus $20 \mu \mathrm{L}$ of cross-linking mixture, $34 \mathrm{mg}$ of KCl, $13 \mathrm{mg}$ of $\mathrm{AgCl}$ (with traces of $\mathrm{Ag}$ ) and $5 \mathrm{mg}$ of ETH500. Twenty microliters of the photo-polymerised nBA or MMA:nBA reference cocktails were drop-cast onto the GC/SWCNTs electrodes, which were then placed upside-down. Photo-polymerisation was carried out using a UV lamp (4 W) for 8 min under argon flow.

Potentiometric testing of all-solid-state reference electrodes

Potentiometric measurements were performed against a conventional double-junction $\mathrm{Ag} / \mathrm{AgCl} / \mathrm{KCl}(3 \mathrm{M})$ reference electrode (type 6.0729.100, Metrohm AG) containing a $1 \mathrm{M}$ LiOAc electrolyte bridge reference electrode. Electromotive forces (EMF) were measured at room temperature $\left(22 \pm 2{ }^{\circ} \mathrm{C}\right)$ using a EMF 16 multichannel data 
acquisition setup and software (Lawson Laboratories, Inc.). The pump systems 700 Dosino and 711 Liquino (Metrohm AG) were used to obtain sequential calibrations from $10^{-1}$ to $10^{-7} \mathrm{M}$ for $\mathrm{KCl}, \mathrm{NaCl}$ and $\mathrm{NaNO}_{3}$. pH sensitivity measurements were performed in a $0.1 \mathrm{M} \mathrm{KCl}$ background solution using a Crison MM40 $\mathrm{pH}$ meter. To measure light sensitivity, the potentiometric signal was first recorded in the dark, then in room light and finally in the dark using an opaque box. Response time is calculated as the time needed to attain the $95 \%$ of the final response $\left(t_{95 \%}\right)$. All data reported was calculated with three electrodes.

\section{Polymer characterisation}

The four polymers described above were characterised by differential scanning calorimetry (DSC) using a Mettler DSC-821e differential scanning calorimeter. The samples weighing between 5 and $10 \mathrm{mg}$ were placed in covered $\mathrm{Al}$ pans under $\mathrm{N}_{2}$, to obtain the DSC curves, which in turn provided glass transition temperatures $\left(T_{\mathrm{g}}\right)$. The copolymers synthesised were also examined by proton nuclear magnetic resonance (NMR). NMR studies were carried out with a $400 \mathrm{MHz}$ VARIAN Mercury VX400 NMR instrument. Samples were dissolved in deuterated chloroform, and the chemical shifts from 0 to $10 \mathrm{ppm}$ were recorded. NMR spectra were used to calculate the cross-linked monomer ratios (Table 1) and polymer purity.

\section{Detection of water percolation}

In order to rapidly detect the percolation of water through the polymeric membranes, we used a $\mathrm{Cu}$ rod instead of the $\mathrm{GC}$ rod. The distal ends of the $\mathrm{Cu}$ rods $(3 \mathrm{~mm} \varnothing)$ were exposed to a $16 \mathrm{~V}$ DC electric field in a distilled water bath to obtain a homogeneous $\mathrm{Cu} / \mathrm{Cu}^{2+}$ surface. Once the $\mathrm{Cu} / \mathrm{Cu}^{2+}$ surface was dry, $100 \mu \mathrm{L}$ of each of the four reference membranes was deposited, letting it dry the necessary time for each membrane. Finally, the electrode was immersed in $3 \mathrm{M}$ or $0.01 \mathrm{M} \mathrm{KCl}$ aqueous solutions, and the percolation of water through the polymeric membranes was assessed by direct observation of the $\mathrm{Cu}_{2} \mathrm{Cl}(\mathrm{OH})_{3}$ on the $\mathrm{Cu}$ rod surface over $24 \mathrm{~h}$. The observable green species were characterised as $\mathrm{Cu}_{2} \mathrm{Cl}(\mathrm{OH})_{3}$ with a Bruker D8 Discover X-Ray diffraction instrument. Blank experiments performed in the absence of $\mathrm{Cl}^{-}$salts or $\mathrm{Cu} / \mathrm{Cu}^{2+}$ surface direct water exposure demonstrated that $\mathrm{Cu}_{2} \mathrm{Cl}(\mathrm{OH})_{3}$ was only formed when the $\mathrm{Cu} / \mathrm{Cu}^{2+}$ surface was exposed to $\mathrm{Cl}^{-}$ anions together with $\mathrm{H}_{2} \mathrm{O}$ molecules.

\section{Results and discussion}

Solid-state reference electrodes based on SWCNTs

SWCNTs have been used here for the first time in a SSRE so the advantageous properties of SWCNTs (i.e. SWCNTs are hydrophobic, and the stable potential created is not affected by UV/Vis light [21]) are exploited to improve the analytical performance of previously reported SSREs. Two different technologies merge in the present SSREs: (1) SWCNTs as excellent ion-to-electron transducer which promote signal stability [21] and (2) polyacrylic membranes that encapsulate $\mathrm{Ag}, \mathrm{AgCl}$ and $\mathrm{KCl}$ salts, creating a constant and reproducible potential [16]. To compare the transduction ability of SWCNTs with CPs, we initially developed SSREs with SWCNTs and photo-nBA reference membrane to test whether the analytical performance of the new SWCNT-based SSREs is comparable to previously reported polypyrrole/photo-nBA-based SSREs [16].

The SSREs were initially characterised by recording their electrode potential following their first contact with a $\mathrm{KCl} 3 \mathrm{M}$ conditioning solution. Twenty-four-hour conditioning was found to be crucial for the stability of photonBA SSREs. The sensitivity of photo-nBA-based SSREs was assessed by recording the potentiometric signal when the types of anion $\left(\mathrm{Cl}^{-}\right.$or $\left.\mathrm{NO}_{3}{ }^{-}\right)$, cation $\left(\mathrm{Na}^{+}\right.$or $\left.\mathrm{K}^{+}\right)$and salt concentration were varied in the test solution. The calibration plots obtained with photo-nBA SSREs are shown in Fig. 2: The recorded slopes were $0.7 \pm 1.7 \mathrm{mV} / \mathrm{dec}$ for $\mathrm{KCl}, 1.0 \pm$ $0.2 \mathrm{mV} / \mathrm{dec}$ for $\mathrm{NaCl}$ and $0.5 \pm 0.4 \mathrm{mV} / \mathrm{dec}$ for $\mathrm{NaNO}_{3}$. Response times $\left(t_{95 \%}\right)$ varied from $1 \mathrm{~min}$ at low concentration to less than $30 \mathrm{~s}$ for higher than $10^{-5} \mathrm{M}$ concentrations. Medium-term stability was recorded in $0.01 \mathrm{M} \mathrm{KCl}$ solution, obtaining a drift of $-1.1 \pm 0.2 \mathrm{mV} / \mathrm{h}$ (Fig. 3, red line). Next,

Table 1 Studied physical characteristics of the polyacrylic polymers

\begin{tabular}{|c|c|c|c|c|}
\hline & $T_{\mathrm{g}}\left({ }^{\circ} \mathrm{C}\right)$ & $\begin{array}{l}\text { Monomer feed ratio } \\
\text { MMA:nBA }\end{array}$ & $\begin{array}{l}\text { Final monomer ratio } \\
\text { MMA:nBA }{ }^{\mathrm{a}}\end{array}$ & $\begin{array}{l}\text { Polymer physical } \\
\text { properties }\end{array}$ \\
\hline Thermo-nBA & -51.5 & $0: 10$ & $0: 10$ & Soft and sticky \\
\hline Thermo-MMA:nBA & -42.6 & $1.1: 10$ & $1.2: 10$ & Elastic and tacky \\
\hline Photo-nBA & -50.4 & $0: 10$ & $0: 10$ & Rubbery \\
\hline Photo-MMA:nBA & -46.1 & $1.1: 10$ & $0.5: 10$ & Rubbery \\
\hline
\end{tabular}

${ }^{\mathrm{a}}$ Determined by NMR 
Fig. 2 Potentiometric response of SWCNT-based photo-nBA SSREs to subsequent calibrations for $\mathrm{KCl}$ (green), $\mathrm{NaCl}$ (red) and $\mathrm{NaNO}_{3}$ (blue) from $10^{-1}$ to $10^{-7} \mathrm{M}$ (logarithmic values are displayed above each segment)

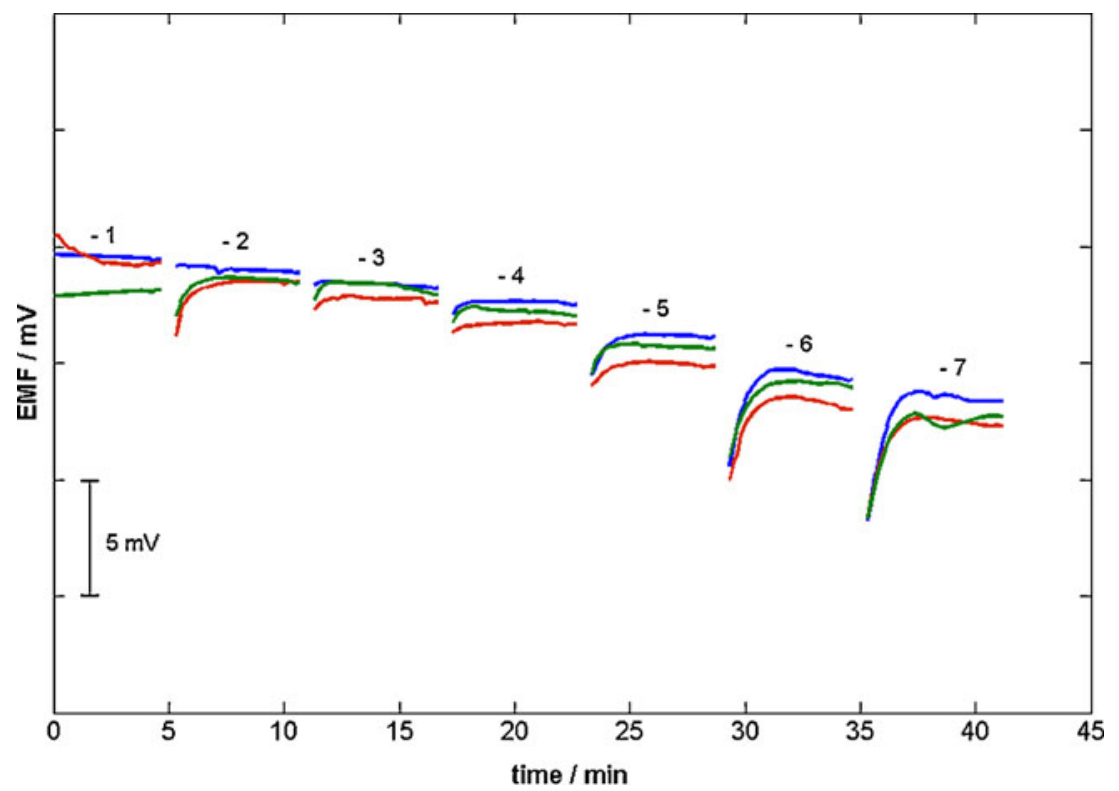

$\mathrm{pH}$ and light variations have been studied in order to determine to what extent they may affect the potential of the SSRE when measuring real samples in in situ analysis. SSREs based on SWCNTs and photo-nBA show no significant variations in potential from $\mathrm{pH} 5$ to 11 (Fig. 4, red dots) and in the presence/absence of room light (Fig. 5, red line).

In the light of the experimental results obtained, we conclude that photo-nBA-based SSREs perform to a similar level with both SWCNTs and polypyrrole. The first two columns in Table 2 present the potentiometric performance characteristics of the SWCNTs and polypyrrole-based SSREs using photo-nBA as reference membrane matrix. Sensitivity to $\mathrm{KCl}, \mathrm{NaCl}, \mathrm{NaNO}_{3}$ and $\mathrm{pH}$ are comparable for SWCNTs and polypyrrole-based SSREs. Only medium-term stability was reported to be lower for POT-based SSREs $(0.2 \mathrm{mV} / \mathrm{h})$, although it should be taken into account that this value was obtained in a more concentrated solution $(0.1 \mathrm{M} \mathrm{KCl})$. What is more, we show here for the first time the insensitivity of a novel SSRE to light exposure, which provides an advantage over other SSREs since light insensitivity is a key aspect in on-field potentiometric measurements.

Knowing that a SWCNTs layer is an excellent solid transducer for this kind of SSRE, we went a step further by investigating the fabrication of SWCNTs-based SSREs with other polyacrylic reference membranes. By doing this, we wanted to (1) develop a SSREs fabricated with polyacrylic membranes which have been already used in solid-state
Fig. 3 Medium-term stability of SWCNTs-based photo-nBA (red) and thermo-MMA:nBA (blue) SSREs in $10^{-2} \mathrm{M} \mathrm{KCl}$

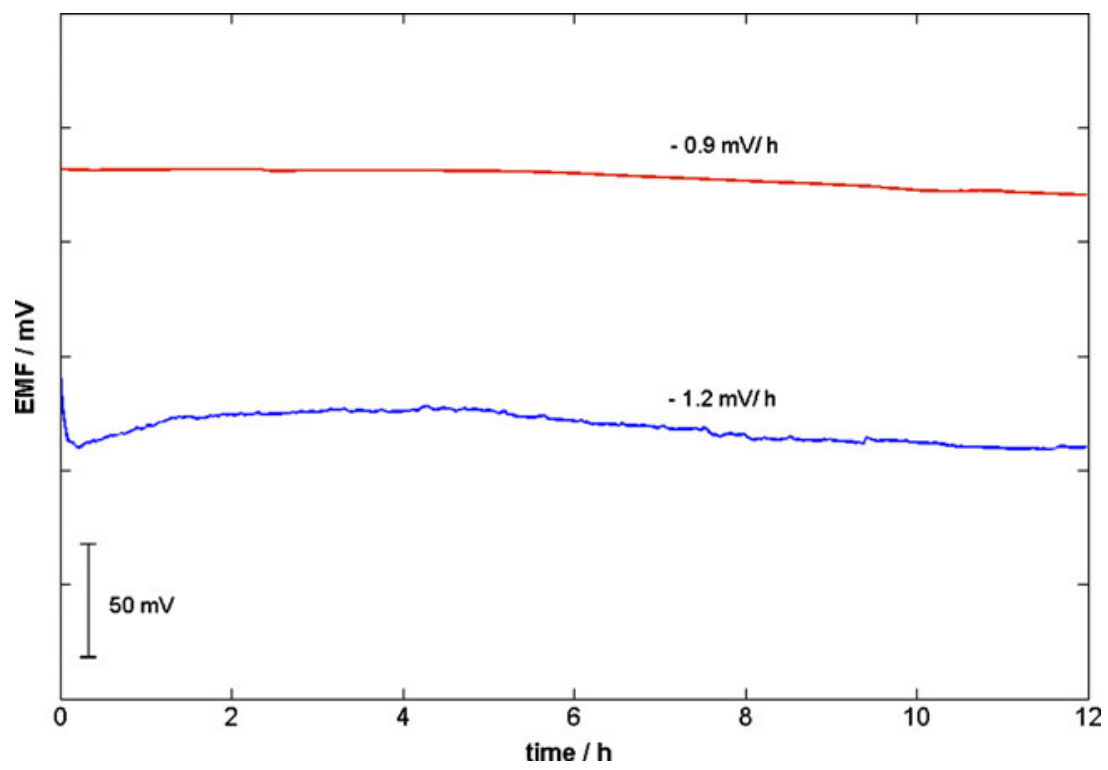


Fig. $4 \mathrm{pH}$ response of

SWCNTs-based photo-nBA

(red) or thermo-MMA:nBA

(blue) SSREs in $10^{-2} \mathrm{M} \mathrm{KCl}$

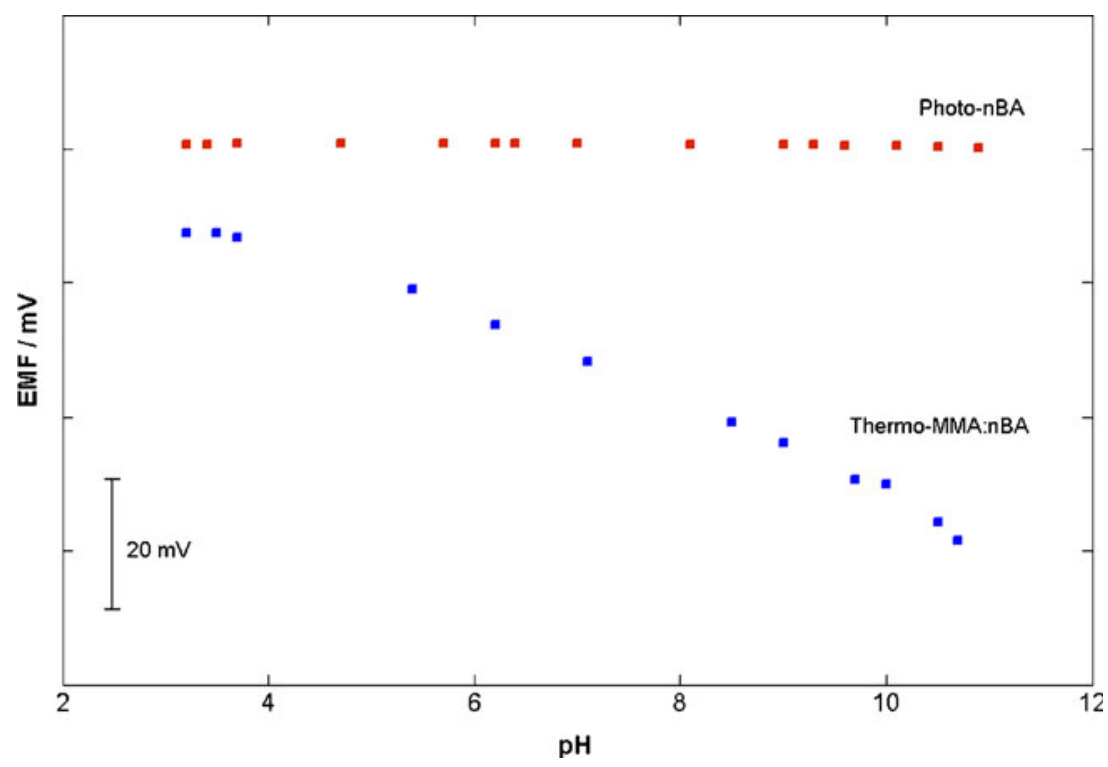

ISEs [28-31] and (2) to obtain more insights on the behaviour of polyacrylic polymers as reservoirs for the $\mathrm{Ag} / \mathrm{AgCl}$ system. We initially selected four polymers for this study: thermo-nBA, thermo-MMA:nBA, photo-nBA and photo-MMA:nBA. These polyacrylic polymers are either produced by UV photocuring or by thermic synthesis and incorporating in their matrix variable amounts of MMA and nBA monomers.

Selecting polyacrylic polymers for SWCNTs-based solid-state reference electrodes

In previous articles, Kisiel et al. used photo-polymerised and thermo-polymerised poly-nBA as membrane materials in SSREs [16, 18]. They found that the physical properties of the polyacrylate polymer influenced the performance of the reference membranes [18]. In the present study, the physical properties of thermo-nBA, thermoMMA:nBA, photo-nBA and photo-MMA:nBA were compared by evaluating their $T_{\mathrm{g}}$ and monomer composition. To our knowledge, the comparison between these four widely used polyacrylic polymers has not been reported before.

Table 1 summarises all the results obtained in the characterisation of the four polyacrylic polymers. The $T_{\mathrm{g}}$ of a polymer is the temperature at which the transition from flexible rubber state to rigid solid (glass) occurs. The molecular chains of polymers with low $T_{\mathrm{g}}$ become flexible
Fig. 5 Light sensitivity of SWCNTs-based photo-nBA (red) containing $\mathrm{Ag}, \mathrm{AgCl}$ and $\mathrm{KCl}$ salts; thermo-MMA:nBA with (dark blue) and without $\mathrm{AgCl}$ (light blue) SSREs

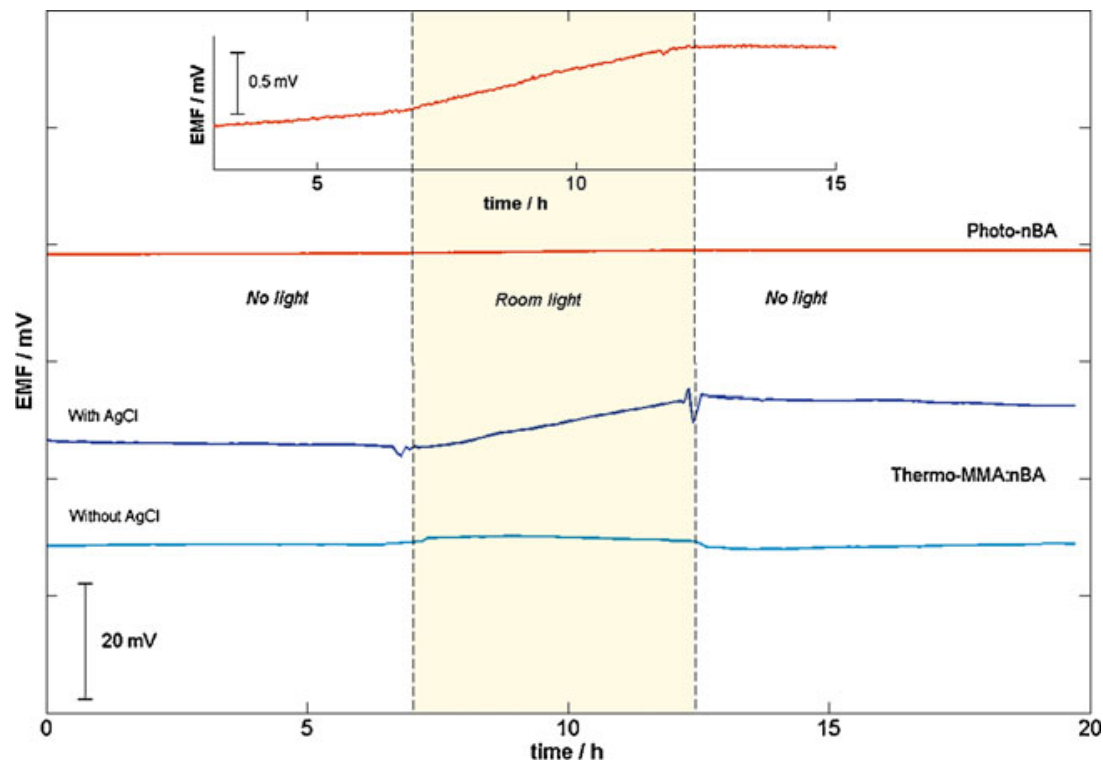


Table 2 Potentiometric performance characteristics of SSREs using polymeric reservoir matrices for the $\mathrm{Ag} / \mathrm{AgCl}$ system and using SWCNTs or CPs as solid-state transducers $[10,16,18]$

\begin{tabular}{|c|c|c|c|c|c|c|c|}
\hline \multicolumn{2}{|l|}{$\begin{array}{l}\text { Polymeric reservoir matrix } \\
\text { Transducer }\end{array}$} & Photo-nBA & Photo-nBA ${ }^{\text {a }}$ & Thermo-MMA.nBA & $\begin{array}{l}\text { Thermo-nBA } \\
\text { POT }\end{array}$ & $\begin{array}{l}\text { PVC-DOS }^{a} \\
\text { PPy(PSS })\end{array}$ & $\begin{array}{l}\text { PVC-DOS } \\
\text { PEDOT(PSS) }\end{array}$ \\
\hline \multicolumn{2}{|l|}{$\begin{array}{l}\text { Conditioning protocol } \\
\text { (conditioning solution/time) }\end{array}$} & $3 \mathrm{M} \mathrm{KCl} / 24 \mathrm{~h}$ & $3 \mathrm{M} \mathrm{KCl} / 12 \mathrm{~h}$ & $\begin{array}{l}3 \mathrm{M} \mathrm{KCl} / 12 \mathrm{~h}+10^{-2} \mathrm{M} \\
\mathrm{KCl} / 12 \mathrm{~h}\end{array}$ & $3 \mathrm{M} \mathrm{KCl} / 48 \mathrm{~h}$ & $3 \mathrm{M} \mathrm{KCl} / 12 \mathrm{~h}$ & $3 \mathrm{M} \mathrm{KCl} / 12 \mathrm{~h}$ \\
\hline \multicolumn{2}{|l|}{$\begin{array}{l}\text { Mid-term stability in } \\
10^{-2} \mathrm{M} \mathrm{KCl}(\mathrm{mV} / \mathrm{h})\end{array}$} & $-1.1 \pm 0.2$ & $0.2^{\mathrm{c}}$ & $-1.2 \pm 0.4$ & nd & nd & nd \\
\hline \multirow[t]{3}{*}{ Sensitivity $(\mathrm{mV} / \mathrm{dec} \log \mathrm{a})^{\mathrm{b}}$} & $\mathrm{KCl}$ & $0.7 \pm 1.7$ & $0.7 \pm 0.1$ & $0.1 \pm 0.6$ & 1 & 0.1 & -0.1 \\
\hline & $\mathrm{NaCl}$ & $1.0 \pm 0.2$ & $0.9 \pm 0.1$ & $0.1 \pm 1.1$ & 0 & 0.7 & -0.2 \\
\hline & $\mathrm{NaNO}_{3}$ & $0.5 \pm 0.4$ & $0.9 \pm 0.2$ & $-0.3 \pm 1.0$ & -1 & 0 & 0.4 \\
\hline Sensitivity (mV/pH unit) ${ }^{\mathrm{d}}$ & $\mathrm{pH}$ & $-0.1 \pm 0.1$ & 0.0 & $-6 \pm 1$ & nd & $1^{\mathrm{e}}$ & nd \\
\hline Sensitivity (mV/min) & Light & $0.1 \pm 0.1$ & nd & $0.6 \pm 0.2$ & nd & nd & nd \\
\hline
\end{tabular}

\footnotetext{
${ }^{\mathrm{a}}$ For the concentration range between 0.1 and $10^{-5} \mathrm{M}$

${ }^{\mathrm{b}}$ For the concentration range between 0.1 and $10^{-7} \mathrm{M}$

${ }^{\mathrm{c}}$ Thermo-nBA microspheres composite. Overnight measurement carried out in $0.1 \mathrm{M} \mathrm{KCl}$

${ }^{\mathrm{d}}$ For the $\mathrm{pH}$ range between 3 and 10

${ }^{\mathrm{e}}$ For the $\mathrm{pH}$ range between 2 and 6
}

enough at room temperature to allow mobility of the electrolytes within the matrix, which is necessary in reference membranes. DSC results clearly show that when MMA monomer is added to the polymer chain the regularity decreases, which in turn increases the $T_{\mathrm{g}}$. Similar results were obtained in previous studies with polyacrylate polymers [32, 33]. Monomer copolymer ratios were studied by NMR spectroscopy [32], which showed that the incorporation of MMA monomers into the final polymeric membrane was favoured in thermo-polymerisation but was hindered for photo-polymerisation. The NMR results agree with the DSC results: the higher the content of polymerised MMA, the more positive the $T_{\mathrm{g}}$.

The four reference membranes used in this work are heterogeneous and contain a high amount of hydrophilic salts, so it is expected that molecular water can enter the bulk membrane and form water islands or channels when it encounters the hydrophilic surfaces of the dispersed salts. Previous studies have studied water uptake in PVC and polyacrylic membranes by several approaches [34-38]. However, these methods for detecting water uptake are cumbersome or not feasible in heterogeneous reference membranes. Therefore, a simple, relatively fast and visual screening test has been used here for the first time to compare the water percolation of the four types of reference membranes. This test is based on the oxidation of $\mathrm{Cu}$ to $\mathrm{Cu}^{2+}$ and its subsequent complexation with $\mathrm{Cl}^{-}$ions and $\mathrm{H}_{2} \mathrm{O}$, which forms green $\mathrm{Cu}_{2} \mathrm{Cl}(\mathrm{OH})_{3}$. Visual detection of green $\mathrm{Cu}_{2} \mathrm{Cl}(\mathrm{OH})_{3}$ indicates the presence of a water layer at the reference membrane/ $\mathrm{Cu}$ interface. Reference membrane cocktails were prepared with the four polymers, deposited onto bare $\mathrm{Cu}$ electrode bodies and immersed in $3 \mathrm{M} \mathrm{KCl}$ solutions. After overnight exposure, green $\mathrm{Cu}_{2} \mathrm{Cl}(\mathrm{OH})_{3}$ were clearly observed in the thermo-nBA, the thermoMMA:nBA and the photo-MMA:nBA polymer membranes. However, $\mathrm{Cu}_{2} \mathrm{Cl}(\mathrm{OH})_{3}$ was not observed in photonBA electrodes. Moreover, it was observed that water percolation also changed the volume and physical properties of the photo-nBA membrane, that is, the photo-nBA reference membrane swelled and partially lost its hydrophobic characteristics. Visual observations indicate that photo-nBA membrane is less prone to water percolation and promotes the accumulation of water molecules surrounding the salt grains. In contrast, the addition of MMA in the polymer chain and also the thermal synthesis produce membranes that did not swell, which suggests that they allow water molecules to be transported through the macromolecular structure of the polymers [13].

Taking the polymer's characterisation results into consideration, thermo-MMA:nBA appears to be an appealing polymer for the fabrication of SWCNTs-based SSREs: thermo-MMA: nBA presents $T_{\mathrm{g}}$, physical properties (see Table 1), water percolation and salt distribution in its bulk that are significantly different from those already studied photo-nBA. In addition, thermo-MMA:nBA has been successfully used in SWCNTs-based ISEs [21, 28-31], so we know that its mechanical properties are compatible with SWCNTs, and it would be of great interest to be able to fabricate both the solid-state ISE and RE with the same polymeric matrix and fabrication procedure. We waived a further study of other membranes due to the following reasons: photo-MMA:nBA physical properties are very similar those of photo-nBA (see Table 1), so it would not add any significant novelty. On the other hand, thermo-nBA has already been studied to 
determine its ability to entrap $\mathrm{Ag} / \mathrm{AgCl}$ and $\mathrm{KCl}$ salts in SSREs [18]: its softness and looser physical properties caused excessive salt leaching from the bulk membrane to the solution when dispersed salts were used, so only when using microspheres to entrap hydrophilic salts the SSRE showed acceptable insensitivity and stability.

\section{Solid-state reference electrode based on SWCNTS and thermo-MMA:nBA membrane}

We first studied the conditioning protocol of the newly tested polymeric membrane: conditioning in $3 \mathrm{M} \mathrm{KCl}$ for $14 \mathrm{~h}$ yielded sensitivities to $\mathrm{KCl}, \mathrm{NaCl}$ and $\mathrm{NaNO}_{3}$ close to $3 \mathrm{mV} / \mathrm{dec}$ and a time of response of 4 min (curve $\mathrm{A}$ in Fig. 6). A shorter time of response was obtained after $14 \mathrm{~h}$ in a $3 \mathrm{M} \mathrm{KCl}$ solution, followed by $14 \mathrm{~h}$ in a $0.01 \mathrm{M} \mathrm{KCl}$ solution. These were the best conditions, and thermo-MMA: nBA SSREs produced the following results: slopes of $0.1 \pm$ $0.6 \mathrm{mV} / \mathrm{dec}$ for $\mathrm{KCl}, 0.1 \pm 1.1 \mathrm{mV} / \mathrm{dec}$ for $\mathrm{NaCl}$ and $-0.3 \pm$ $1.0 \mathrm{mV} / \mathrm{dec}$ for $\mathrm{NaNO}_{3}$ for concentration from $10^{-1}$ to $10^{-7} \mathrm{M}$ (curve B in Fig. 6). Short response times ( $t_{95} \%$ less than $30 \mathrm{~s}$ ) were obtained over the whole operational range. Figure 3 shows the medium-term stability $(12 \mathrm{~h}$ experiment) of the SWCNTs-based thermo-MMA:nBA SSRE, pointing to an initial positive drift followed by a signal plateau and a slight negative drift of $-1.2 \pm 0.4 \mathrm{mV} / \mathrm{h}$.

Since thermo-MMA:nBA membranes allow for intense water percolation, the thermo-MMA:nBA SSREs were further studied by keeping the electrodes in dry conditions for 1 week. During this time, water content inside the membrane is expected to significantly decrease. When we recorded the potentiometric response to variations in salt concentration immediately after being kept in dry conditions, the thermo-MMA:nBA SSREs showed drifts and long response times $(4 \mathrm{~min})$. The results improved after exposing the thermo-MMA:nBA SSRE to optimal conditioning described above: sensitivities to $\mathrm{KCl}, \mathrm{NaCl}$ and $\mathrm{NaNO}_{3}$ were then below $2 \mathrm{mV} / \mathrm{dec}$, with times of response of $1 \mathrm{~min}$ and satisfactory medium-term stability $(2 \mathrm{mV} / \mathrm{h}$; see curve $\mathrm{C}$ in Fig. 6). Only a shift of $20 \mathrm{mV}$ for the starting potentials of $\mathrm{K}^{+}$and $\mathrm{Na}^{+}$salts differentiated the results of thermo-MMA:nBA SSREs after 1 week storage in dry conditions. The latter results were promising, suggesting that it is possible to storage the thermo-MMA:nBA for a long time in dry conditions without maintenance, and only an appropriate conditioning protocol is needed to yield insensitivity to $\mathrm{KCl}, \mathrm{NaCl}$ and $\mathrm{NaNO}_{3}$.

Thermo-MMA.nBA showed a linear response of $-6 \pm$ $1 \mathrm{mV} / \mathrm{pH}$ unit from $\mathrm{pH} 3$ to 11 (see Fig. 4, blue dots). The varying dispersion of the salts in the bulk membrane, the different membrane lipophilicity and different water percolation through the membrane can cause the response to changes in $\mathrm{pH}$. Thermo-MMA:nBA SSREs showed practically no drift in dark conditions; however, when the potentiometric cell was exposed to daylight, a constant lineal drift of $0.6 \pm 0.2 \mathrm{mV} / \mathrm{min}$ appeared (see Fig. 5, dark blue line). When $\mathrm{AgCl}$ was removed from the reference thermo-MMA:nBA membrane, we observed only a $1 \mathrm{mV}$ jump of potential when the potentiometric cell was exposed to daylight (see Fig. 5, light blue line). These results corroborate that $\mathrm{AgCl}$ is the agent causing light sensitivity. The photovoltaic effect forces the thermo-MMA:nBA SSRE to be integrated into dark cartridges to protect it from changes in ambient light.
Fig. 6 Potentiometric response of SWCNT-based thermoMMA:nBA SSRE to subsequent calibrations for $\mathrm{KCl}$ (green), $\mathrm{NaCl}$ (red) and $\mathrm{NaNO}_{3}$ (blue) from $10^{-1}$ to $10^{-7} \mathrm{M}$ (logarithmic values are displayed above each segment): $(A)$ conditioned in $3 \mathrm{M} \mathrm{KCl}$ for $14 \mathrm{~h} ;(B)$ conditioned in $3 \mathrm{M} \mathrm{KCl}$ for $14 \mathrm{~h}$ and $10^{-2} \mathrm{M} \mathrm{KCl}$ for $14 \mathrm{~h} ;(C)$ after 1 week kept dry and conditioned in $0.01 \mathrm{M} \mathrm{KCl}$ for $14 \mathrm{~h}$

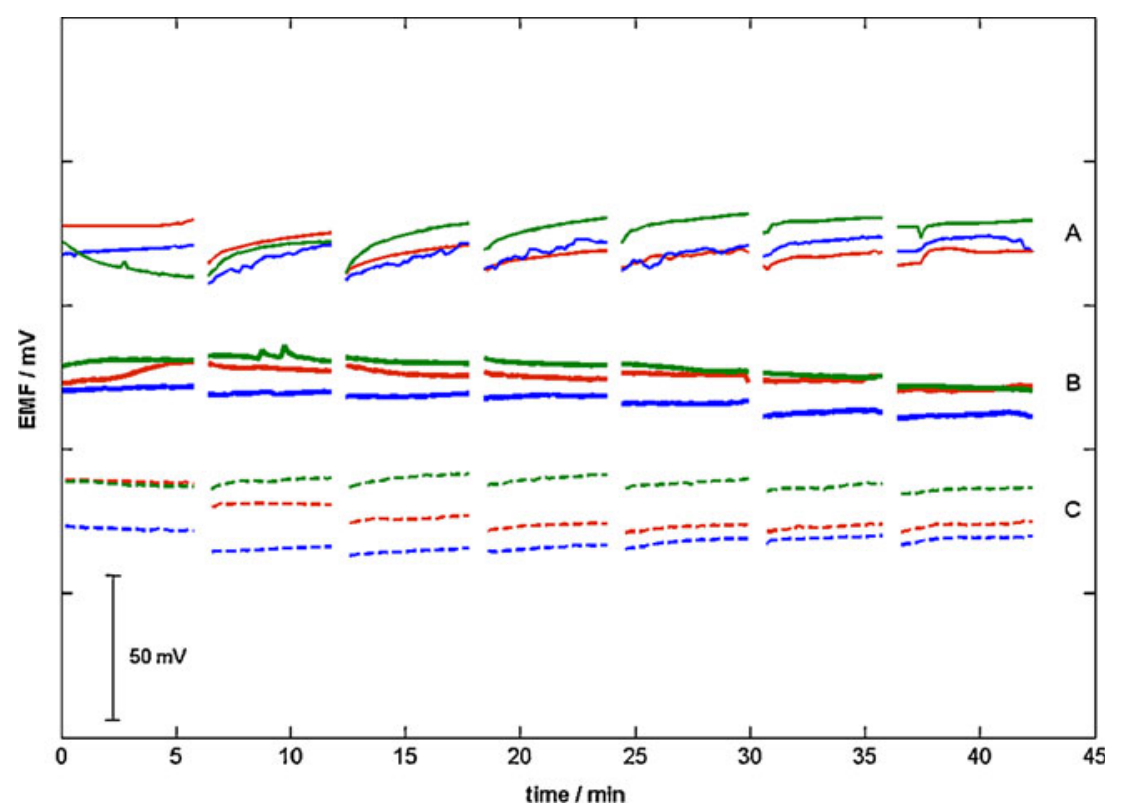




\section{Role of SWCNTs and polyacrylic reference membranes}

In order to study the role of SWCNTs as efficient transducer of the potentiometric signal, we have compared the analytical parameters found for SWCNTs-based SSREs (i.e. using photo-nBA and thermo-MMA:nBA membranes) with comparable studies previously reported on CPs-based SSREs by Kisiel et al. [10, 16, 18] (see Table 2). SWCNTs-based SSREs show comparable analytical performance values in terms of $\mathrm{KCl}, \mathrm{NaCl}, \mathrm{NaNO}_{3}$ and $\mathrm{pH}$ insensitivity. Reported mid-term stability values for polypyrrole/photo-nBA-based SSREs are lower than for SWCNTs/photo-nBA SSREs, although one should take into account that mid-term stability can vary depending on solution concentration and stirring conditions. In this article, we report for the first time on a SWCNTs/photo-nBA SSRE being insensitive to light exposure. In short, we show that SWCNTs are a suitable transducer for SSREs, especially for on-field applications.

The role of the silver/silver chloride system contained in the reference membrane was investigated by removing the silver chloride and silver ion from the thermo-MMA:nBA reference membranes. It was found that reference membranes without $\mathrm{AgCl}$ responded to salt concentration variations. In another experiment, $\mathrm{Ag} / \mathrm{AgCl}$ and $\mathrm{KCl}$ salts were all removed from the thermo-MMA:nBA reference membrane, while the membrane electroneutrality was assured following the method by Mattinnen et al. [6]: it was observed that electrodes in their first contact with water had no response to low $\mathrm{KCl}$ concentration $\left(10^{-7}\right.$ to $\left.10^{-4} \mathrm{M}\right)$, but signal started drifting at high $\mathrm{KCl}$ concentrations $\left(10^{-4}\right.$ to $\left.10^{-2} \mathrm{M}\right)$. After overnight conditioning in $10^{-3} \mathrm{M} \mathrm{KCl}$, electrodes showed response to $\mathrm{KCl}$ concentration variations. These results show that thermo-MMA:nBA membrane is permeable to ions, and these ions can interfere in the potential stability of the SSRE. Therefore, it is clear that dispersed $\mathrm{Ag} / \mathrm{AgCl}$ and $\mathrm{KCl}$ salts play an active role in maintaining a stable membrane potential.

Besides, photo-nBA and thermo-MMA:nBA polymers were proved to act as efficient solid reservoirs. Insensitivity to changes in the sample electrolyte concentration was observed after 1 month dry storage for both photo-nBA and thermo-MMA:nBA; insensitivity to $\mathrm{KCl}, \mathrm{NaCl}$ and $\mathrm{NaNO}_{3}$ never exceeded $2 \mathrm{mV} / \mathrm{dec}$, results that are not significantly different from those shown in Figs. 2 and 6 for photo-nBA and thermo-MMA:nBA SWCNTs-based SSREs, respectively.

\section{Conclusions}

A new set of SSREs has been developed using SWCNTs as the ion-to-electron transducer and polyacrylic polymers as the $\mathrm{Ag} / \mathrm{AgCl}$ system reservoir. SWCNTs transducer layer is shown to be comparable, and in some cases superior, to other solid transducers for the fabrication of SSREs. The best results were obtained with photo-nBA reference membrane SWCNTs-based SSRE: satisfactory medium-term stability and insensitivity to changes in $\mathrm{KCl}, \mathrm{NaCl}, \mathrm{NaNO}_{3}$ and $\mathrm{pH}$ was observed. Besides, it did not respond to changes in room light, which has not been reported before for similar SSREs. Newly applied thermo-MMA:nBA showed response to $\mathrm{pH}$ and light in SWCNTs-based SSRE. New techniques (such as encapsulating components within polyacrylate microspheres [18]) would add value to this first study on the use of thermo-MMA:nBA in SWCNTs-based SSREs.

The high water percolation through the reference membranes has been qualitatively assessed, and it was found that thermo-MMA:nBA is more prone to water percolation than photo-nBA. This is in agreement with DSC and NMR results: photo-nBA is less prone to water percolation because it is a more ordered polymer $\left(T_{\mathrm{g}}=-50.4{ }^{\circ} \mathrm{C}\right)$, while the addition of $10 \%$ MMA monomer to the poly-nBA chain decreases the order of the polyacrylate matrix $\left(T_{\mathrm{g}}=-42.6{ }^{\circ} \mathrm{C}\right)$.

Polyacrylic membranes have been shown to be effective solid reservoirs, so it is possible to storage the SWCNTsbased SSREs for a long time in dry conditions without maintenance, and only proposed conditioning protocols have to be followed to obtain the reported insensitivity values. This new type of SWCNTs-based SSRE is simple to fabricate and miniaturise since it only contains SWCNTs as the transducer layer and a polyacrylic polymer to entrap the $\mathrm{Ag} / \mathrm{AgCl}$ system. Moreover, using the same polyacrylic polymer in both the SSRE and the ISE simplifies the process of fabricating the entire potentiometric cell.

Acknowledgments We thank the Spanish Ministry of Science and Innovation (MICINN) for supporting this work under project grant CTQ2010-18717 and Polish scientific research funds within the research project N204 242234. FXR-R also thanks the Universitat Rovira i Virgili for the economic support given.

Open Access This article is distributed under the terms of the Creative Commons Attribution Noncommercial License which permits any noncommercial use, distribution, and reproduction in any medium, provided the original author(s) and source are credited.

\section{References}

1. Bakker E, Pretsch E (2007) Modern potentiometry. Angew Chem Int Ed 46(30):5660-5668

2. Vincze A, Horvai G (1997) The design of reference electrode without liquid junction. Electrochem Soc Proc 97(19):550

3. Bakker E (1999) Hydrophobic membranes as liquid junction-free reference electrodes. Electroanalysis 11(10-11):788-792

4. Lee HJ, Hong US, Lee DK, Shin JH, Nam H, Cha GS (1998) Solvent-processible polymer membrane-based liquid junction-free reference electrode. Anal Chem 70(16):3377-3383

5. Maminska R, Dybko A, Wróblewski W (2006) All-solid-state miniaturised planar reference electrodes based on ionic liquids. Sens Actuators B 115(1):552-557 
6. Mattinen U, Bobacka J, Lewenstam A (2009) Solid-contact reference electrodes based on lipophilic salts. Electroanalysis 21 (17-18):1955-1960

7. Mangold KM, Schäfer S, Jüttner K (2001) Reference electrodes based on conducting polymer bilayers. Synt Metals 119(1-3):345346

8. Blaz T, Migdalski J, Lewenstam A (2005) Junction-less reference electrode for potentiometric measurements obtained by buffering ph in a conducting polymer matrix. Analyst 130(5):637-643

9. Ghilane J, Hapiot P, Bard AJ (2006) Metal/polypyrrole quasireference electrode for voltammetry in nonaqueous and aqueous solutions. Anal Chem 78(19):6868-6872

10. Kisiel A, Marcisz H, Michalska A, Maksymiuk K (2005) Allsolid-state reference electrodes based on conducting polymers. Analyst 130(12):1655-1662

11. Lindfors $T$ (2009) Light sensitivity and potential stability of electrically conducting polymers commonly used in solid contact ion-selective electrodes. J Solid State Electrochem 13(1):77-89

12. Guth U, Gerlach F, Decker M, Oelßner W, Vonau W (2009) Solidstate reference electrodes for potentiometric sensors. J Solid State Electrochem 13(1):27-39

13. Tymecki L, Zwierkowska E, Koncki R (2004) Screen-printed reference electrodes for potentiometric measurements. Anal Chim Acta 526(1):3-11

14. Kwon N-H, Lee K-S, Won M-S, Shim Y-B (2007) An all-solidstate reference electrode based on the layer-by-layer polymer coating. Analyst 132(9):906-912

15. Kakiuchi T, Yoshimatsu T, Nishi N (2007) New class of Ag/AgCl electrodes based on hydrophobic ionic liquid saturated with $\mathrm{AgCl}$. Anal Chem 79(18):7187-7191

16. Kisiel A, Michalska A, Maksymiuk K, Hall EAH (2008) Allsolid-state reference electrodes with poly(n-butyl acrylate) based membranes. Electroanalysis 20(3):318-323

17. Kisiel A, Michalska A, Maksymiuk K (2007) Plastic reference electrodes and plastic potentiometric cells with dispersion cast poly(3,4-ethylenedioxythiophene) and poly(vinyl chloride) based membranes. Bioelectrochem 71(1):75-80

18. Kisiel A, Donten M, Mieczkowski J, Rius-Ruiz FX, Maksymiuk K, Michalska A (2010) Polyacrylate microspheres composite for all-solid-state reference electrodes. Analyst 135(9):2420-2425

19. Vonau W, Oelßner W, Guth U, Henze J (2010) An all-solid-state reference electrode. Sens Actuators B 144(2):368-373

20. Düzgün A, Zelada-Guillén G, Crespo GA, Macho S, Riu J, Rius FX (2011) Nanostructured materials in potentiometry. Anal Bioanal Chem 399(1):171-181

21. Crespo GA, Macho S, Rius FX (2008) Ion-selective electrodes using carbon nanotubes as ion-to-electron transducers. Anal Chem 80(4):1316-1322

22. Crespo GA, Macho S, Bobacka J, Rius FX (2009) Transduction mechanism of carbon nanotubes in solid-contact ion-selective electrodes. Anal Chem 81(2):676-681

23. Yáñez-Sedeño P, Pingarrón JM, Riu J, Rius FX (2010) Electrochemical sensing based on carbon nanotubes. TrAC Trends Anal Chem 29(9):939-953
24. Anastasova-Ivanova S, Mattinen U, Radu A, Bobacka J, Lewenstam A, Migdalski J, Danielewski M, Diamond D (2010) Development of miniature all-solid-state potentiometric sensing system. Sens Actuators B 146(1):199-205

25. Michalska AJ, Appaih-Kusi C, Heng LY, Walkiewicz S, Hall EAH (2004) An experimental study of membrane materials and inner contacting layers for ion-selective $\mathrm{K}^{+}$electrodes with a stable response and good dynamic range. Anal Chem 76:2031-2039

26. Furtado CA, Kim UJ, Gutierrez HR, Pan L, Dickey EC, Eklund PC (2004) Debundling and dissolution of single-walled carbon nanotubes in amide solvents. J Am Chem Soc 126(19):6095-6105

27. Michalska A, Wojciechowski M, Bulska E, Mieczkowski J, Maksymiuk K (2009) Poly(n-butyl acrylate) based lead (II) selective electrodes. Talanta 79(5):1247-1251

28. Ampurdanés J, Crespo GA, Maroto A, Sarmentero MA, Ballester P, Rius FX (2009) Determination of choline and derivatives with a solid-contact ion-selective electrode based on octaamide cavitand and carbon nanotubes. Biosens Bioelectron 25(2):344-349

29. Parra EJ, Crespo GA, Riu J, Ruiz A, Rius FX (2009) Ion-selective electrodes using multi-walled carbon nanotubes as ion-to-electron transducers for the detection of perchlorate. Analyst 134(9):1905-1910

30. Hernandez R, Riu J, Rius FX (2010) Determination of calcium ion in sap using carbon nanotube-based ion-selective electrodes. Analyst 135(8):1979-1985

31. Crespo GA, Gugsa D, Macho S, Rius FX (2009) Solid-contact phselective electrode using multi-walled carbon nanotubes. Anal Bioanal Chem 395(7):2371-2376

32. Heng LY, Hall EAH (2000) Methacrylic-acrylic polymers in ionselective membranes: achieving the right polymer recipe. Anal Chim Acta 403(1-2):77-89

33. Malinowska E, Gawart L, Parzuchowski P, Rokicki G, Brzózka Z (2000) Novel approach of immobilization of calix[4]arene type ionophore in 'self-plasticized' polymeric membrane. Anal Chim Acta 421(1):93-101

34. Fibbioli M, Morf WE, Badertscher MF, de Rooij N, Pretsch E (2000) Potential drifts of solid-contacted ion-selective electrodes due to zero-current ion fluxes through the sensor membrane. Electroanalysis 12(16):1286-1292

35. Appiah-Kusi C, Kew SJ, Hall EAH (2009) Water transport in poly (n-butyl acrylate) ion-selective membranes. Electroanalysis 21 $(17-18): 1992-2003$

36. Sundfors F, Lindfors T, Höfler L, Bereczki R, Gyurcsányi RE (2009) FTIR-ATR study of water uptake and diffusion through ion-selective membranes based on poly(acrylates) and silicone rubber. Anal Chem 81(14):5925-5934

37. Veder J-P, Patel K, Clarke G, Grygolowicz-Pawlak E, Silvester DS, De Marco R, Pretsch E, Bakker E (2010) Synchrotron radiation/Fourier transform-infrared microspectroscopy study of undesirable water inclusions in solid-contact polymeric ionselective electrodes. Anal Chem 82(14):6203-6207

38. Veder JP, De Marco R, Clarke G, Chester R, Nelson A, Prince K, Pretsch E, Bakker E (2008) Elimination of undesirable water layers in solid-contact polymeric ion-selective electrodes. Anal Chem 80(17):6731-6740 